
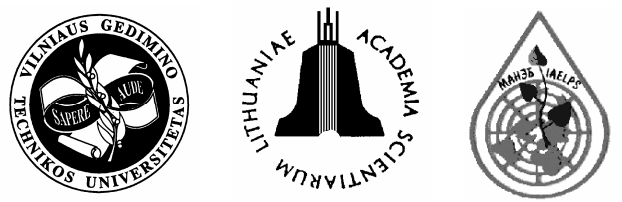

\title{
EXTENSIVE USE OF SOWN MEADOWS - A TOOL FOR RESTORATION OF BOTANICAL DIVERSITY
}

\author{
Jūratė Sendžikaitė, Romas Pakalnis \\ Laboratory of Landscape Ecology, Institute of Botany, Žaliuju Ežeru g. 49, 08406 Vilnius, Lithuania. \\ E-mail: jursend@botanika.lt
}

Submitted 30 Mar 2006; accepted 16 Apr 2006

\begin{abstract}
The state of sown meadow communities of different intensity of management (intensive and extensive using) over the period of 10-14 years of running was evaluated at Graisupis Experimental Field Station, Lithuania. Comparison of study data on intensively and extensively used sown meadows enabled to ascertain that intensity of sown meadows succession depends upon the character of grassland management. The positive correlation between the number of vascular plant species and sown meadow age revealed that botanical diversity of species in extensively used grassland restores much faster $\left(r_{E}=0,95\right)$ than under intensive management conditions $\left(r_{I}=0,59\right)$.
\end{abstract}

Keywords: sown meadow, succession, extensive and intensive use, productivity, botanical diversity, restoration.

\section{Introduction}

Meadows in the forest zone are mostly synanthropic, i e they occur because of human impact upon the primary landscape (instead of cleared, burnt forests or reclaimed bogs) of course, with the exception of natural wetlands and flood meadows [1-3]. As the forests were cleared, grasslands were established under the influence of domestic stock by a process of natural recruitment of grasses and herbs from forest glades and wetlands. These grassžlands varied in their structure and floristic composition according to the prevailing ecological conditions and the intensity of management [2]. Under temperate climate conditions meadow ecosystems are unstable and considered as an intermediate stage of succession of plant communities. Communities of semi-natural meadows formed as a result of centuries-long traditions of land use, which determined the formation of meadow communities characterised by high species diversity of vascular plants. In the second half of the 20th century, however, intensification of agricultural activities (land reclamation, enlargement of arable land areas, excessive fertilisation) in Central and East Europe inevitably affected the whole natural environment. The changes unavoidably influenced meadow ecosystems, especially those valuable in ecological aspect but less important economically. The survival not only of natural (even flooded) but also seminatural meadows and preservation of their biological diversity is threatened. Only at the end of the 20th century, as the status of land property and farming priorities was changed and the need for fodder decreased, the process of cultural grasslands naturalization and meadow restoration started: the structure of communities, productivity, economic value of grasslands change, and the di- versity of plant species and even meadow communities increases [4].

Presently, investigations on communities of natural and sown meadows become particularly important. Researchers worldwide are engaged in the issues of meadow succession and restoration [3, 5-11]. For the research on the development of meadow communities, it is essential to monitor and evaluate the condition of the natural and sown meadows in Lithuania, predict their changes, leave the areas of the necessary size to ensure the preservation of biological diversity, and to choose an appropriate regime of their maintenance. As Lithuania has become a Member State of the EU, there appear new possibilities to preserve botanical diversity of the communities in the remaining natural and restoring semi-natural meadows. The financial assistance to farmers for well maintained meadows encourage them to take care, mow, and prevent the overgrowth with shrubs. Regarding the preservation of botanical diversity, extensive farming involving minimum financial expenditure, refusal or only minimal fertilization and mowing of the meadow at least once per season would be very efficient.

The aim of the investigation is to evaluate changes in the communities of naturalizing intensively and extensively used sown meadows and possibilities for restoration of botanical diversity.

\section{Material and methods}

The object of the study was intensively and extensively used sown mesophillous meadow communities undergoing the process of naturalization.

Methods. The status of intensively and extensively used sown meadow communities was evaluated in the 
period of 2001-2005 (June-August) at the Experimental Field Station (EFS) of Graisupis (Kèdainiai district; $\mathrm{N} 55^{\circ} 19^{\prime}-20^{\prime}$, E $\left.23^{\circ} 49^{\prime}-50^{\prime}\right)$, representing the region of the Central Lithuanian Plain. To establish the station, a grassland mixture $(27 \mathrm{~kg} / \mathrm{ha}$; Festuca pratense, Dactylis glomerata, Poa pratensis, Phleum pratense, Lolium perenne, Trifolium pratense, and T. repens) was sown into the arable field in 1991 [12]. Intensively used grassland is being fertilized, hay made and pastured every year, extensively used grassland is not fertilized, but it is undergoing yearly haymaking and rare pasturage. The grassland develops on plain landscape, the relief of which does not have strong influence on differences in ecological conditions of ecosystems, therefore, human activity and meteorological conditions in growth season are decisive factors.

Flora composition of the grasslands and aboveground phytomass (dry weight, $\mathrm{g} / \mathrm{m}^{2}$ ) were registered in June (1st harvest), July (2nd harvest) and August (3rd harvest) on two experimental field sites (intensively and extensively used). On every experimental field site, three permanent study plots $\left(100 \mathrm{~m}^{2}\right)$ were singled out each divided into three trial plots of $1 \mathrm{~m}^{2}$ in size (Fig 1).

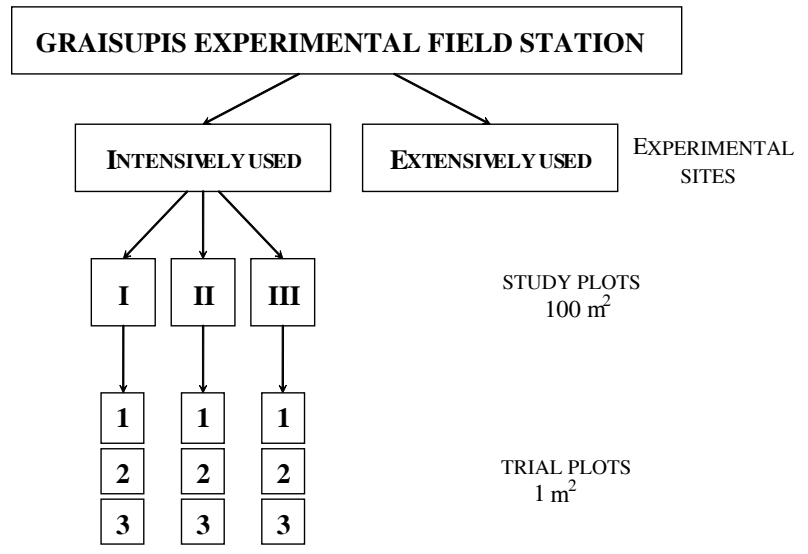

Fig 1. Scheme of Graisupis Experimental Field Station

Phytosociological relevés were made and aboveground phytomass was estimated in the same permanent places. Phytocenotic descriptions of sown meadow communities were performed applying the principles of vegetation research of French-Swiss (Zürich-Montpelier) school [13-14]. Abundance of species of sown meadow communities and herb layer coverage were evaluated according to J. Braun-Blanquet [13] scale on the study plots $\left(100 \mathrm{~m}^{2}\right)$. On individual experimental sites the frequency $(\%)$ of every vascular plant species was ascertained. Table 1 presents general data on sown meadow flora composition at Graisupis EFS. Nomenclature of plant species was applied following Z. Gudžinskas [15].

Aboveground phytomass of meadow communities was ascertained according to the programme and methods of geobotanical investigations [16-17]. Three times per growth season (1st-3rd harvests) on each study plot the aboveground phytomass was determined on three typical (by flora composition and herb layer coverage) trial plots of $1 \mathrm{~m}^{2}$ in size. The aboveground part, mown down to the soil level, was grouped into vascular plants (arranged into species), bryophytes and dead parts of plants. The sorted out sample was dried up and weighed, i e the specific weight of every grass species was indicated (Table 2). Economic value of the communities was indicated following the methods of A. Petkevičius and A. Stancevičius [18]. Ecological groups of vascular plants were presented by means of H. Ellenberg [19] scale.

Similarity of meadow communities was ascertained according to Sörensen coefficients: $\mathrm{C}_{\mathrm{S}}-$ to compare communities according to flora composition of the recorded vascular plants, $\mathrm{C}_{\mathrm{N}}$ - to compare communities according to aboveground phytomass of vascular plants [20].

The data were processed applying Microsoft Excel and STATISTICA for Windows software. Correlation coefficient ( $\mathrm{r}$ ) was used to figure interrelation of diverse features [21].

\section{Natural conditions}

According to physical and geographical division of Lithuania, the area of Graisupis EFS is situated on the Central Lithuanian Plain [21-22]. Graisupis EFS is located on the right side of the Nevėžis River basin between the rivulets Jaugila and Smilga. The area has been reclaimed intensively. Among natural perennial vegetation distribution ranges, only larger agricultural forest of Ažzuolaičiai (78,6 ha) and some small agricultural forests have survived. The agricultural area of the basin includes a continuous territory of arable land and sown meadows (the area of individual distribution ranges exceeds 200 ha). The landscape of undulating, ridged loamy plain prevails in this area. Soil forming rocks of morainic or limnoglacial origins occur, light loam with occasional sand threads dominates. Gleyic calcaric cambisols prevail at the EFS of Graisupis, like on the whole Central Lithuanian Plain because of carbon-bearing parent rocks (carbonates are washed out at a depth of 40-60 cm) [22-25].

\section{Results}

The condition of sown meadow communities of different intensity of management (intensive and extensive using) over the period of 10-14 years of running was evaluated (Figs 2, 3).

In an intensively used meadow, during five years of investigation, 39 vascular plant species were recorded; in separate years the number varied from 22 (in 2002) to 30 (in 2005). Among them 6 Poaceae (Dactylis glomerata, Festuca pratensis, Lolium perenne, Phleum pratense, Poa pratensis, P. annua), 3 Fabaceae (Trifolium repens, T. pratense, T. hybridum) and 30 species (Taraxacum officinale, Leontodon autumnalis, Achillea millefolium, Alchemilla vulgaris, Capsella bursa-pastoris, Cerastium holosteoides, Cirsium arvense, C. vulgare, Matricaria discoidea, Plantago lanceolata, Potentilla anserina, Rumex crispus, etc) of other families were identified; with an average reaching 13-20 species per $100 \mathrm{~m}^{2}$ of study plots (Table 1, Figs 4, 5).

Grassland of high and very high economic value $(8,0-9,1$ points) is of average productivity - 720$1340 \mathrm{~g} / \mathrm{m}^{2}$ of aboveground phytomass (Table 2, Figs 6, 7). 


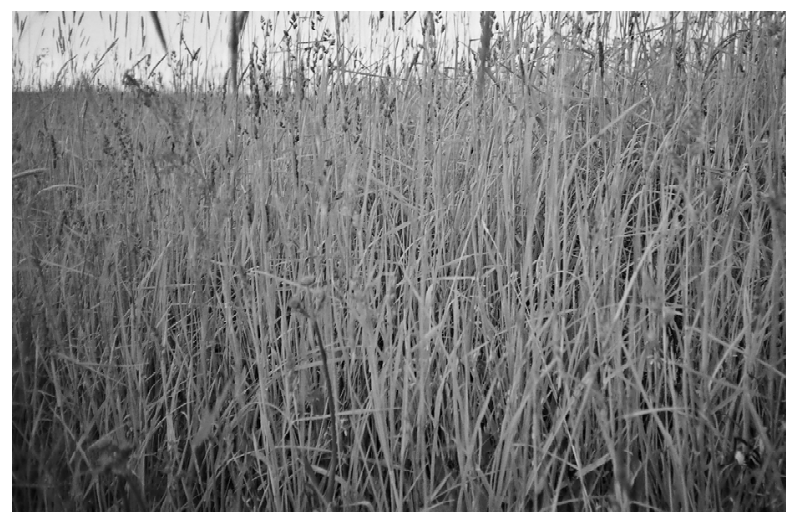

Fig 2. Intensively used sown meadow communities

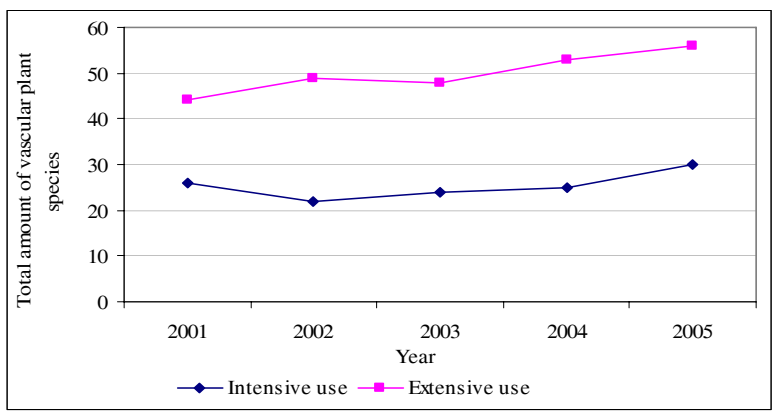

Fig 4. Total amount of vascular plants species in intensively and extensively used sown meadow communities, Graisupis EFS, Lithuania

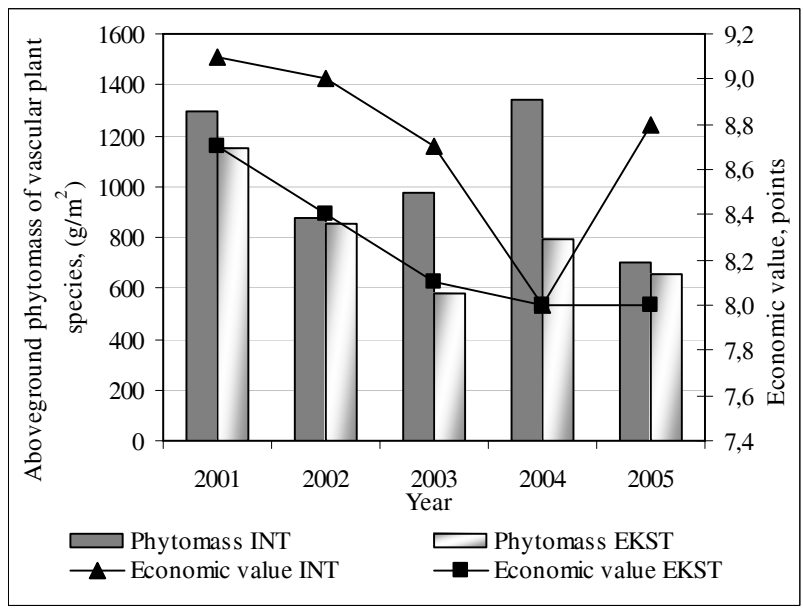

Fig 6. Phytomass (dry weight, $\mathrm{g} / \mathrm{m}^{2}$ ) of vascular plants species and economic value (points) of grasslands in intensively and extensively used sown meadow communities, Graisupis EFS, Lithuania

Intensive use of the grassland predetermines low amount of bryophytes (up to $1,4 \%$ ) and dead parts of plants $(0,8-12,0 \%)$ in the total aboveground phytomass (Table 3 , Fig 7). An annual tendency towards decline of the total aboveground phyto-mass $\left(r_{I}=-0,53\right)$ and phytomass of vascular plants $\left(r_{E}=-0,42\right)$ was noticed (Table 4).

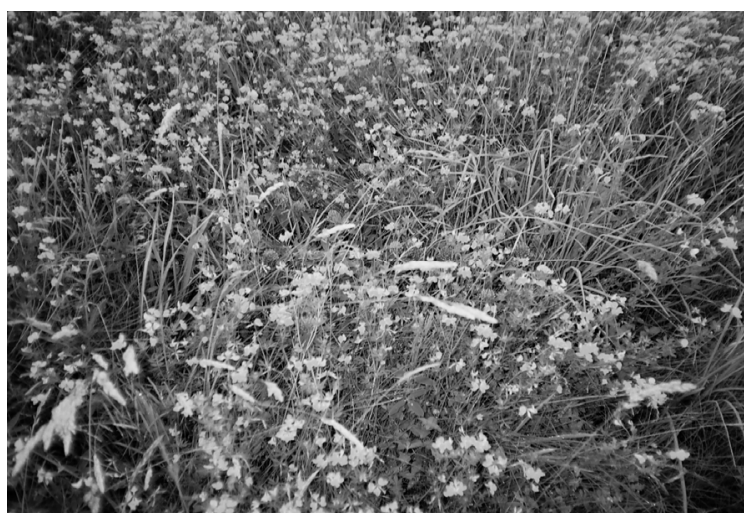

Fig 3. Extensively used sown meadow communities

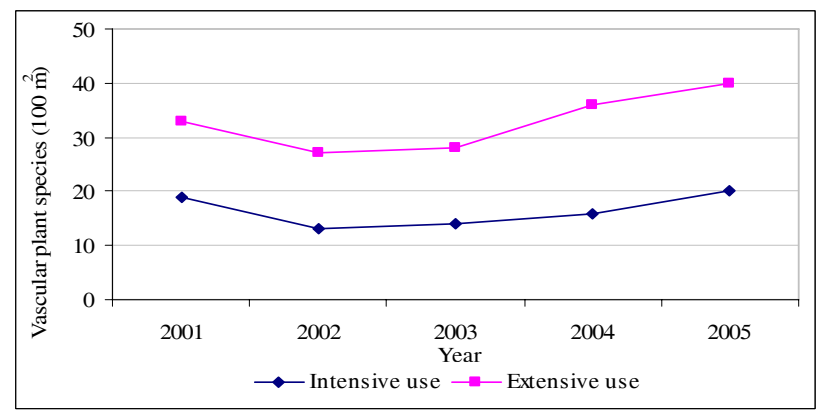

Fig 5. Amount og vascular plants species in intensively and extensively used sown meadow communities $\left(100 \mathrm{~m}^{2}\right)$, Graisupis EFS, Lithuania

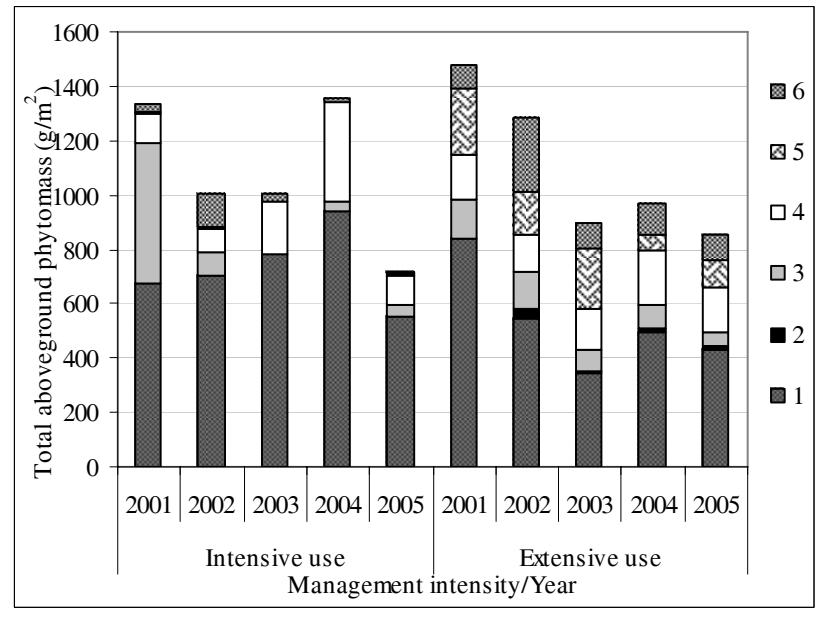

Fig 7. Phytomass (dry weight, $\mathrm{g} / \mathrm{m}^{2}$ ) of the aboveground part of sown meadow communities, Graisupis EFS, Lithuania: 1 - Poaceae; 2 - Cyperaceae and Juncaceae; 3 - Fabaceae; 4- other species; 5- bryophytes, 6 - dead parts of plants

Low diversity of vascular plants is due to intensive meadow use. It confirms the opinion of B. H. Green [2] that botanical diversity of productive meadows restores much slower. In annually fertilized grasslands, the sown species consistent with the existing ecological conditions are more competitive; they get enough nutritious substances; therefore, plants of these species suppress 
Table 1. Sown meadow composition and frequency (\%) of vascular plants species in intensively and extensively used sown meadow communities, Graisupis Experimental Field Station, Kedainiai district, Lithuania, June-August 2001-2005

\begin{tabular}{|c|c|c|c|c|c|c|c|c|c|c|}
\hline \multirow{2}{*}{\begin{tabular}{|l|} 
Experimental sites \\
Year
\end{tabular}} & \multicolumn{5}{|c|}{ Intensive use (I) } & \multicolumn{5}{|c|}{ Extensive use (E) } \\
\hline & 2001 & 2002 & 2003 & 2004 & 2005 & 2001 & 2002 & 2003 & 2004 & 2005 \\
\hline Total number of relevés & 9 & 9 & 9 & 9 & 9 & 9 & 9 & 9 & 9 & 9 \\
\hline \begin{tabular}{|l|l|} 
Coverage (\%) & herb layer \\
\end{tabular} & 70 & 60 & 65 & 90 & 90 & 60 & 65 & 70 & 65 & 60 \\
\hline bryophyte layer & 30 & 10 & 10 & 5 & 5 & 90 & 90 & 90 & 85 & 80 \\
\hline Average number of species & 19 & 13 & 14 & 16 & 20 & 33 & 27 & 28 & 36 & 40 \\
\hline Total number of species & 26 & 22 & 24 & 25 & 30 & 44 & 49 & 48 & 53 & 56 \\
\hline \begin{tabular}{|r|}
1 \\
\end{tabular} & 2 & 3 & 4 & 5 & 6 & 7 & 8 & 9 & 10 & 11 \\
\hline Achillea millefolium & 100 & 100 & 100 & 100 & 100 & 100 & 75 & 83 & 100 & 100 \\
\hline Aegopodium podagraria & . & . & . & . & . & . & . & . & . & 22 \\
\hline Agrostis capillaris & . & . & . & . & & . & . & . & . & 44 \\
\hline Ajuga reptans & . & . & . & . & . & . & 8 & . & . & . \\
\hline Alchemilla vulgaris & . & 17 & 8 & 11 & 44 & 42 & 33 & 50 & 67 & 89 \\
\hline Alnus incana & . & . & . & . & . & 92 & 75 & 8 & 44 & . \\
\hline Angelica sylvestris & . & . & . & . & . & . & 25 & . & . & . \\
\hline Anthemis arvensis & . & . & . & . & . & . & . & . & . & . \\
\hline Anthoxanthum odoratum & . & . & . & . & . & 67 & 33 & 50 & 78 & 89 \\
\hline Anthriscus sylvestris & . & . & . & . & 33 & 8 & 33 & . & 22 & 22 \\
\hline Arctium lappa & 25 & . & . & . & 11 & . & . & . & . & . \\
\hline Artemisia vulgaris & & . & . & 22 & 22 & . & 17 & . & . & . \\
\hline Barbarea vulgaris & 17 & . & . & . & . & . & . & . & . & . \\
\hline Campanula patula & . & . & . & . & . & 33 & 42 & 67 & 100 & 89 \\
\hline Capsella bursa-pastoris & 67 & 33 & 75 & 100 & 89 & . & . & . & . & . \\
\hline Carex hirta & . & . & . & $\cdot$ & . & 42 & 83 & 50 & 56 & 100 \\
\hline Carex panicea & . & . & . & . & . & . & 17 & 25 & 44 & 67 \\
\hline Centaurea jacea & . & . & . & . & . & . & 17 & . & . & . \\
\hline Centaurium erythraea & . & . & . & . & . & 92 & 58 & 67 & 100 & 89 \\
\hline Cerastium holosteoides & 67 & 58 & 17 & 67 & 100 & 92 & . & 25 & 67 & 78 \\
\hline Chenopodium album & & & 8 & & 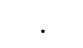 & & . & . & . & . \\
\hline Cirsium arvense & 83 & 75 & 33 & 56 & 100 & 100 & 100 & 75 & 33 & 67 \\
\hline Cirsium palustre & . & . & $\cdot$ & . & . & $\cdot$ & $\cdot$ & . & 11 & 56 \\
\hline Cirsium rivulare & . & . & . & . & . & 25 & 8 & 33 & 44 & 33 \\
\hline Cirsium vulgare & 33 & 33 & 33 & 11 & 100 & 25 & 25 & 17 & 89 & 44 \\
\hline Convolvulus arvensis & 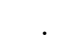 & . & . & . & . & . & $\cdot$ & 8 & . & . \\
\hline Dactylis glomerata & 100 & 100 & 100 & 100 & 100 & 100 & 100 & 100 & 100 & 100 \\
\hline Deschampsia cespitosa & . & . & . & . & . & 100 & 100 & 100 & 100 & 100 \\
\hline Equisetum arvense & . & . & . & . & . & 67 & 33 & . & . & . \\
\hline Erygeron acris & . & . & . & . & . & . & . & . & . & . \\
\hline Euphrasia rostkoviana & 17 & . & . & . & . & . & . & . & . & . \\
\hline Festuca pratensis & 100 & 100 & 100 & 100 & 100 & 100 & 100 & 100 & 100 & 100 \\
\hline Festuca rubra & · & $\cdot$ & . & . & $\cdot$ & . & . & . & . & 78 \\
\hline Fragaria vesca & . & . & . & . & . & . & . & . & . & . \\
\hline Galium mollugo & . & . & . & . & . & . & . & 17 & 22 & . \\
\hline Galium uliginosum & . & . & . & . & . & . & . & . & 44 & . \\
\hline Geum rivale & 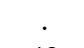 & . & . & . & . & 33 & 33 & 25 & 67 & 100 \\
\hline Glechoma hederacea & 42 & . & . & 11 & 33 & 83 & 33 & 83 & 56 & 100 \\
\hline Heracleum sibiricum & . & . & . & . & . & . & . & . & . & 11 \\
\hline Hypericum maculatum & . & . & . & . & . & 100 & 100 & 75 & 100 & 100 \\
\hline Hypericum perforatum & . & . & . & . & . & . & . & . & . & . \\
\hline Juncus conglomeratus & . & . & . & . & . & 58 & 58 & 42 & 56 & 56 \\
\hline Lathyrus pratensis & $\cdot$ & . & . & . & . & 100 & 100 & 100 & 100 & 100 \\
\hline Leontodon autumnalis & 67 & 58 & 17 & 22 & 44 & 58 & 67 & 42 & 56 & 67 \\
\hline Leontodon hispidus & . & . & . & . & . & . & . & . & 11 & . \\
\hline Leucanthemum vulgare & & . & $\cdot$ & $\cdot$ & $\cdot$ & 92 & 58 & 33 & 78 & 78 \\
\hline Lolium perenne & 100 & 100 & 100 & 100 & 100 & . & . & . & . & . \\
\hline Lotus corniculatus & . & . & . & $\cdot$ & . & 67 & 58 & 33 & 67 & 100 \\
\hline Luzula multiflora & . & . & . & . & . & 75 & 75 & 67 & 100 & 100 \\
\hline Lychnis flos-cuculi & . & . & . & . & . & 83 & 75 & 83 & 100 & 100 \\
\hline Lysimachia nummularia & . & . & . & 11 & . & 100 & 92 & 100 & 100 & 100 \\
\hline
\end{tabular}


Table 1 (continued)

\begin{tabular}{|c|c|c|c|c|c|c|c|c|c|c|}
\hline $\begin{array}{r} \\
\end{array}$ & 2 & 3 & 4 & 5 & 6 & 7 & 8 & 9 & 10 & 11 \\
\hline Matricaria discoidea & . & 83 & 50 & 67 & 78 & 8 & & & & . \\
\hline Medicago lupulina & . & . & . & . & . & . & 8 & 50 & 33 & . \\
\hline Medicago sativa & . & . & . & . & . & . & . & . & . & . \\
\hline Mentha arvensis & . & . & . & . & . & 17 & . & 17 & 22 & 44 \\
\hline Myosotis arvensis & . & . & . & . & . & . & . & . & . & . \\
\hline Myosotis scorpioides & . & . & . & . & . & 83 & 33 & 8 & 33 & 33 \\
\hline Odontites vulgaris & . & . & . & . & . & . & 25 & 17 & 56 & 44 \\
\hline Peucedanum palustre & . & . & . & . & . & . & . & . & . & 44 \\
\hline Phleum pratense & 17 & 67 & 100 & 100 & 100 & 100 & 100 & 100 & 100 & 100 \\
\hline Pilosella officinarum & . & . & . & . & . & . & . & . & . & . \\
\hline Pimpinella saxifraga & . & . & . & . & . & 25 & . & . & 11 & . \\
\hline Plantago lanceolata & 100 & . & 17 & . & 11 & . & . & . & . & . \\
\hline Plantago major & 67 & . & 25 & 11 & 22 & . & . & 25 & . & 11 \\
\hline Pоа аппиа & 83 & 58 & 92 & 100 & 100 & . & . & . & . & . \\
\hline Poa pratensis & 100 & 100 & 100 & 100 & 100 & 100 & 100 & 100 & 100 & 100 \\
\hline Polygonum aviculare & $\cdot$ & . & 33 & . & . & . & . & . & . & . \\
\hline Potentilla anserina & 58 & . & . & 44 & 33 & 100 & 100 & 100 & 100 & 100 \\
\hline Prunella vulgaris & . & . & . & . & . & . & 42 & 17 & 33 & 56 \\
\hline Ranunculus acris & . & . & . & . & . & 83 & 58 & 33 & 67 & 89 \\
\hline Ranunculus repens & . & 33 & . & . & 56 & 100 & 100 & 75 & 67 & 100 \\
\hline Raphanus raphanistrum & 17 & 17 & . & . & . & . & . & . & . & . \\
\hline Rumex acetosa & . & . & . & . & 22 & 42 & 8 & 33 & 67 & 44 \\
\hline Rumex confertus & 83 & 17 & 33 & . & . & . & . & . & . & . \\
\hline Rumex crispus & 83 & 83 & 8 & 100 & 100 & 83 & 58 & 58 & 67 & 56 \\
\hline Salex sp. & . & . & . & . & . & . & . & . & . & 44 \\
\hline Sanguisorba officinalis & . & . & . & . & . & . & . & . & . & 11 \\
\hline Senecio jacobaea & . & . & . & . & . & . & . & . & 56 & . \\
\hline Sonchus arvensis & . & . & . & . & . & . & . & . & . & . \\
\hline Stellaria graminea & . & 17 & . & . & . & . & . & . & 56 & 56 \\
\hline Tanacetum vulgare & . & . & . & . & . & . & . & . & . & 22 \\
\hline Taraxacum officinale & 100 & 100 & 100 & 100 & 100 & 100 & 100 & 100 & 100 & 100 \\
\hline Trifolium hybridum & . & . & . & 44 & 67 & 83 & 75 & 100 & 33 & 89 \\
\hline Trifolium pratense & 100 & 100 & 100 & 78 & 100 & 50 & 33 & 100 & 100 & 78 \\
\hline Trifolium repens & 100 & 75 & 100 & 100 & 100 & 92 & 75 & 100 & 100 & 100 \\
\hline Tripleurospermum perforatum & 50 & . & . & . & . & . & . & . & . & $\cdot$ \\
\hline Tussilago farfara & . & . & . & . & . & . & . & . & . & . \\
\hline Urtica dioica & . & . & . & . & . & . & 8 & . & . & . \\
\hline Veronica chamaedrys & . & . & . & . & 22 & 92 & 75 & 67 & 100 & 67 \\
\hline Veronica serpyllifolia & . & . & . & . & . & . & . & . & . & 33 \\
\hline Vicia angustifolia & . & . & . & . & . & . & . & 58 & 78 & 100 \\
\hline Vicia cracca & . & . & . & . & . & 100 & 100 & 100 & 100 & 100 \\
\hline Viola arvensis & 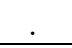 & . & 8 & 22 & 33 & . & . & 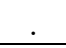 & & . \\
\hline
\end{tabular}

the development of slower growing and less demanding plants. Consequently, fertilizers increase the productivity of grassland but impede the restoration of botanical diversity. According to M. H. Losvik [26] and A. P. Huhta [27], species diversity of vascular plants is strongly related with a negative balance of nutrients in plant habitats. Rather large amounts of nutritious substances are removed while mowing (dry aboveground phytomass is taken away from the meadow) or grazing. The deficiency of nutrients in the habitats of non-fertilized meadows inhibits the growth of sown plants and increases possibilities for new species to establish and botanical diversity to increase.

In extensively used meadows a considerably higher species diversity of vascular plants was recorded. During the investigation, 71 species of vascular plants were registered; an average number of species per study plot was
27-40. Among them 8 Poaceae (Dactylis glomerata, Deschampsia cespitosa, Festuca pratensis, F. rubra, Phleum pratense, Poa pratensis, Anthoxanthum odoratum, Agrostis capillaris), 2 Cyperaceae (Carex hirta, C. panicea) and Juncaceae (Juncus conglomeratus, Luzula multiflora), 8 Fabaceae (Lathyrus pratensis, Lotus corniculatus, Medicago lupulina, Trifolium hybridum, T. pratense, T. repens, Vicia angustifolia, V. cracca) and 51 species of other families were determined (Table 1, Figs 4, 5). Close proximity of forest and minimum farming activities induced quicker succession of the sown grassland than in the case of intensive farming. In the grassland besides valuable and economically important fodder species unvaluable but ecologically significant species, the indicators of meadow naturalization (e $g$ Agrostis capillaris, Anthoxanthum odoratum, Carex hirta, C. panicea, Cirsium rivulare, Deschampsia cespitosa, 


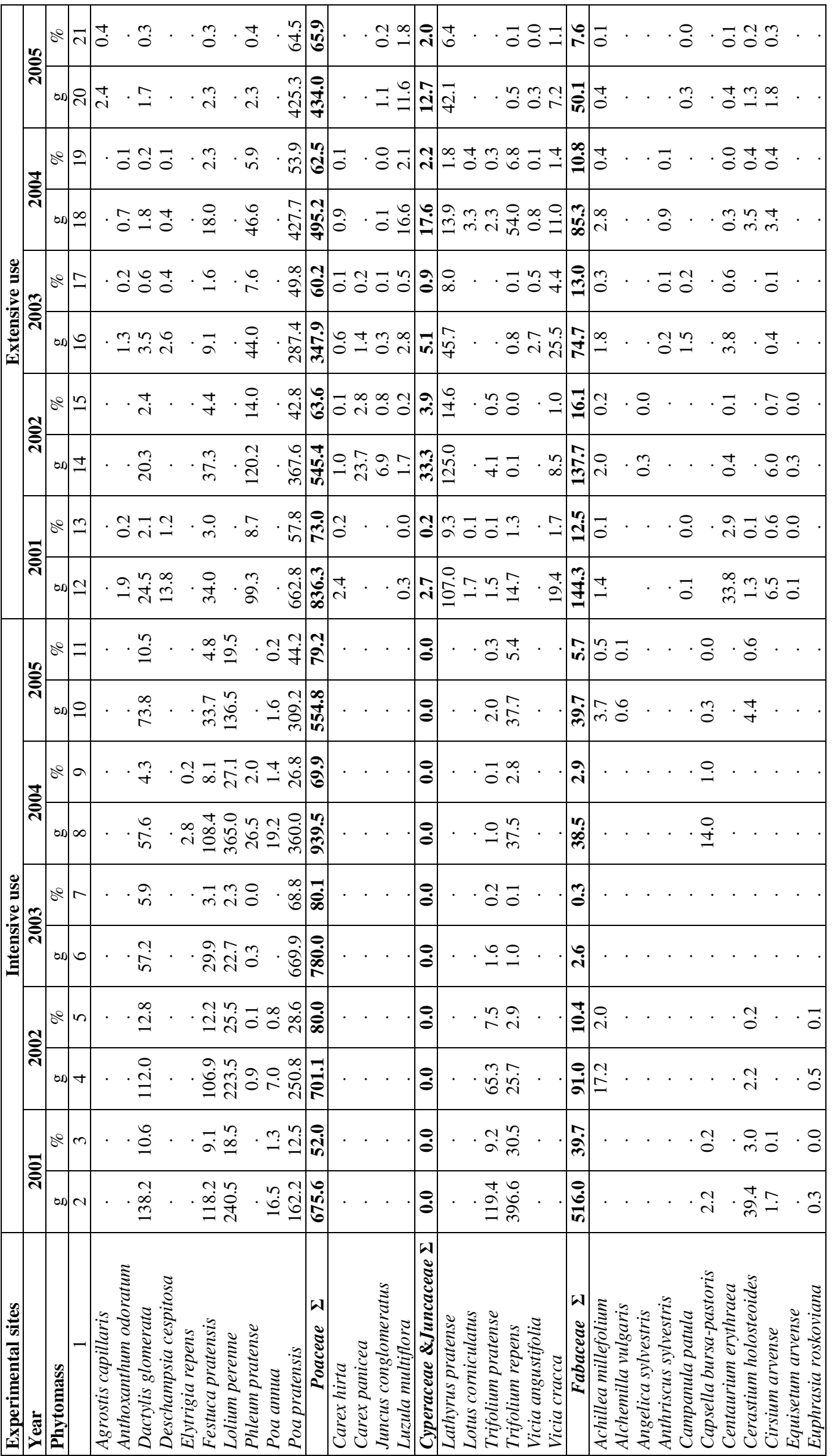




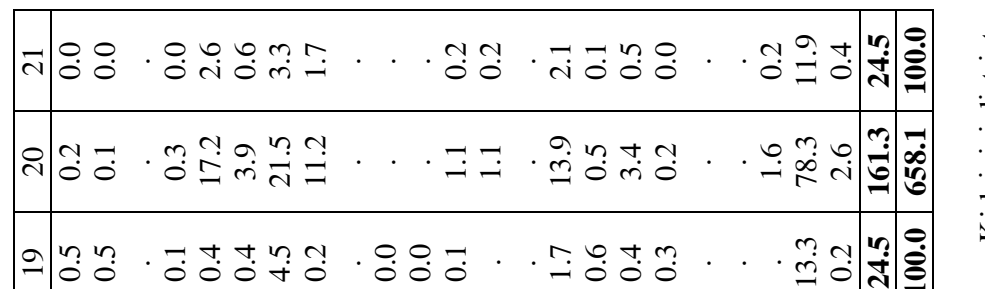

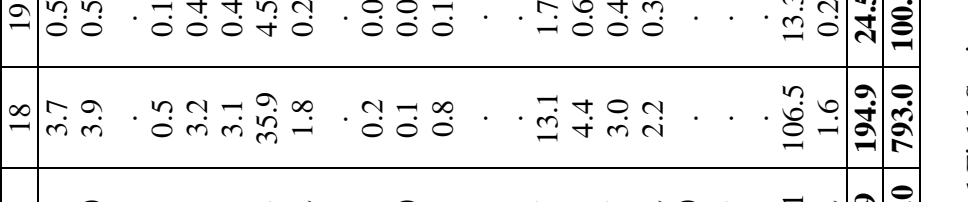

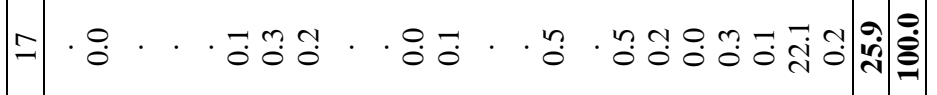

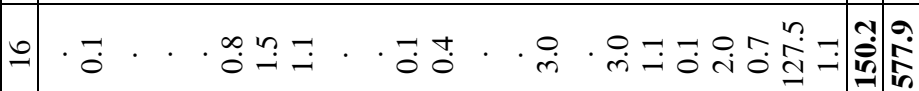

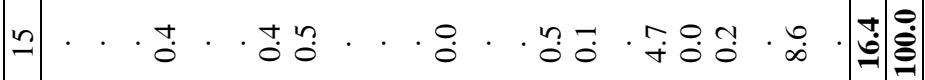

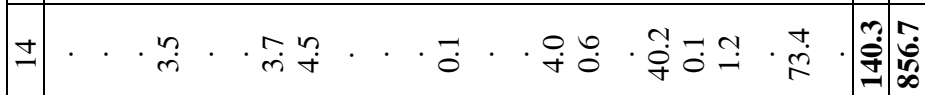

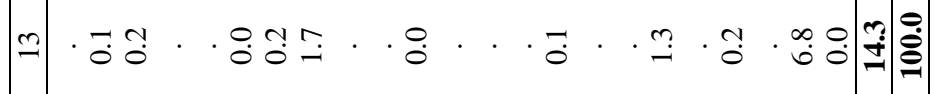

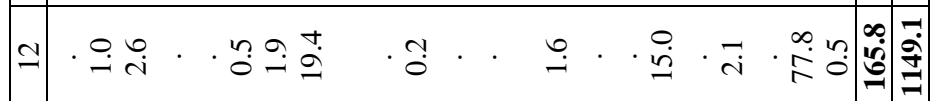

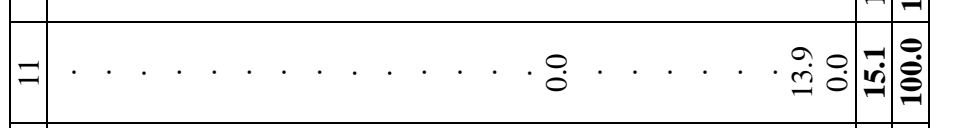

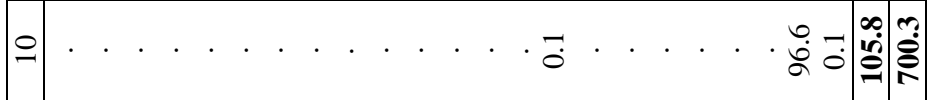

○

$\infty \ldots \ldots \ldots$

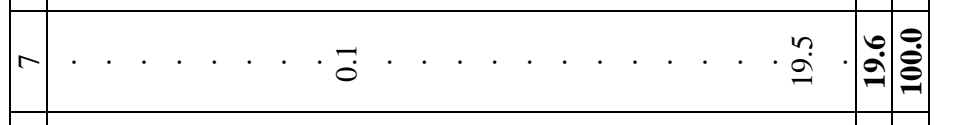

○

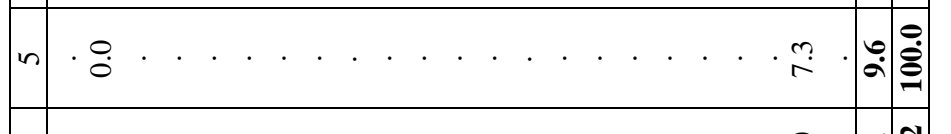

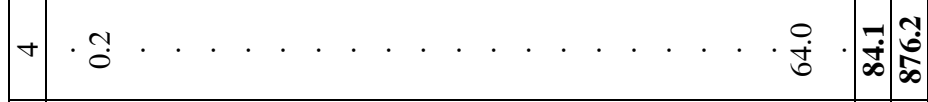

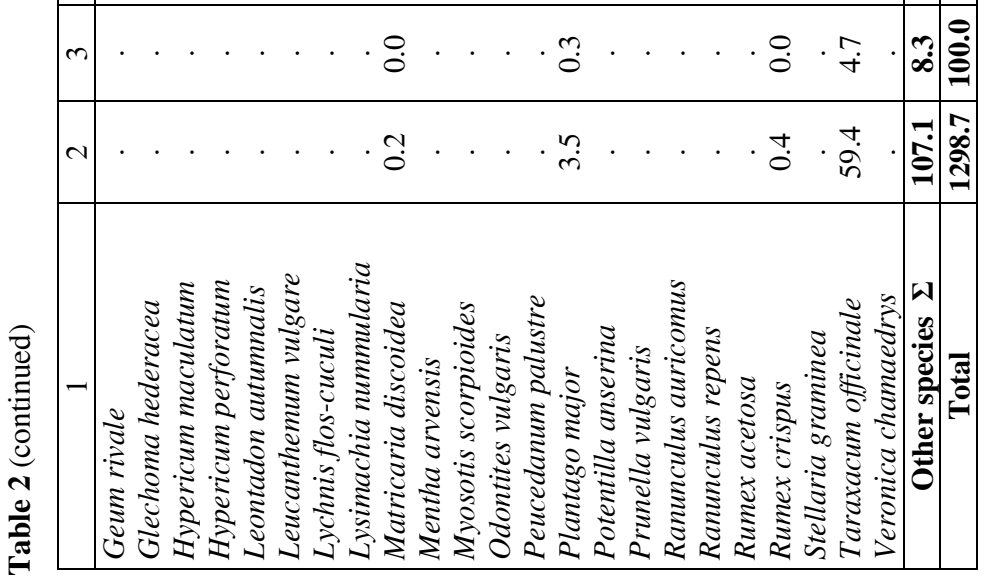

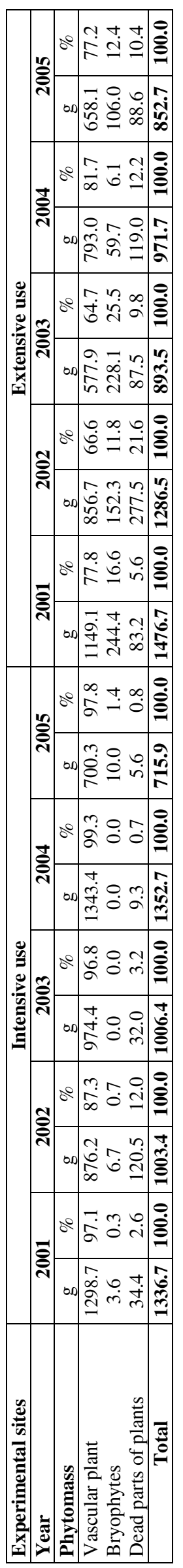


Table 4. Changes in variables of sown meadow quality (by correlation analysis; $r$ - correlation coefficient; character of management: $I$ - intensive use, $E$ - extensive use), Graisupis EFS, Kèdainiai district, Lithuania, 2001-2005

\begin{tabular}{|c|c|c|c|}
\hline \multicolumn{2}{|l|}{ Variables } & \multicolumn{2}{|c|}{ Character of management } \\
\hline$x$ & $y$ & $r_{I}$ & $r_{E}$ \\
\hline Number of vascular plants species & Age of sown meadows & 0,59 & 0,95 \\
\hline Aboveground phytomass of vascular plants $\left(\mathrm{g} / \mathrm{m}^{2}\right)$ & Age of sown meadows & $-0,42$ & $-0,75$ \\
\hline Aboveground phytomass of vascular plants $\left(\mathrm{g} / \mathrm{m}^{2}\right)$ & Number of vascular plants species & $-0,27$ & $-0,63$ \\
\hline Total aboveground phytomass $\left(\mathrm{g} / \mathrm{m}^{2}\right)$ & Age of sown meadows & $-0,53$ & $-0,91$ \\
\hline Total aboveground phytomass $\left(\mathrm{g} / \mathrm{m}^{2}\right)$ & Number of vascular plants species & $-0,40$ & $-0,77$ \\
\hline Economic value of grassland & Age of sown meadows & $-0,59$ & $-0,93$ \\
\hline Economic value of grassland & Number of vascular plants species & $-0,01$ & $-0,85$ \\
\hline
\end{tabular}

Geum rivale, Glechoma hederaceae, Hypericum maculatum, Juncus conglomeratus, Leucanthemum vulgare, Lotus corniculatus, Luzula multiflora, Lychnis floscuculi, Lysimachia nummularia, Mentha arvensis, Myosotis scorpioides, Pimpinella saxifraga, Potentilla anserina, Ranunculus acris, $R$. repens, Vicia angustifolia, etc), are also abundant; their constancy is rather high. Bryophyte coverage reaches $80-90 \%$. The grassland is formed of constantly and abundantly growing mesophytic plants (Dactylis glomerata, Festuca pratensis, Phleum pratense, Poa pratensis, Lathyrus pratensis, Taraxacum officinale).

Grassland of high and very high economic value $(8,0-8,7$ points $)$ is of average productivity - $850-$ $1480 \mathrm{~g} / \mathrm{m}^{2}$ of aboveground phytomass (Figs 6, 7). Extensive use of the grassland conditioned rather large amount of bryophytes $(6,1-25,6 \%)$ and dead parts of plants $(5,6-$ $21,6 \%$ ) (Table 3, Fig 7). During five years of investigation, distinct annual tendency towards the reduction of the total aboveground phytomass $\left(r_{E}=-0,91\right)$ and vascular plant phytomass $\left(r_{E}=-0,75\right)$ was observed (Table 4$)$.

Comparison of the data on botanical diversity and aboveground phytomass weight of intensively and extensively used sown meadows revealed that the character of grassland management influenced the intensity of the succession. During five years of investigation, in extensively used sown meadows a considerably higher number of vascular plant species (71 species) was identified comparing with intensively used grasslands (39 species). A low number ( 23 species) of vascular plant species, inventoried in both extensively and intensively used grasslands (Table 1), determined a low value of Sörensen coefficient $\left(\mathrm{C}_{S}=0,42\right)$. It proves that the investigated meadows undergo different succession stages. The determined positive correlation between the number of vascular plant species and the age of sown meadows $\left(r_{E}=0,95 ; r_{I}=\right.$ $0,59)$ demonstrated that in extensively used grassland the species diversity restored considerably quicker than in intensive used ones (Table 4).

Modified quantitative Sörensen coefficient values obtained by the comparison of grasslands of a different character of management according to the aboveground phytomass produced by plants of each species confirm the impact exerted by the meadow management upon the grassland condition. The coefficient shows that in 2001, $\mathrm{i}$ e on the 10th year of running, the composition of the aboveground phytomass of the compared grasslands clearly differed $\left(C_{N}=0,24\right)$. On the 14 th year of running, however, steady settled Poa pratensis and Taraxacum officinale plants producing $44,2 \%$ and $13,8 \%$ (intensive use), $64,5 \%$ and $11,9 \%$ (extensive use) of aboveground phytomass of vascular plants (Table 2), respectively, conditioned stronger similarity of the compared grasslands $\left(C_{N}=0,58\right)$.

Negative correlation determined between aboveground phytomass of vascular plants and the age of sown meadows $\left(r_{I}=-0,42 ; r_{E}=-0,75\right)$ shows that productivity of meadows of both management types has a tendency to decline; it is particularly evident in the case of extensive use conditions. Analogous relationship is observed regarding an economic value of the grassland and its age (year of meadow use, $r_{I}=-0,59 ; r_{E}=-0,93$ ) as well as the number of vascular plant species $\left(r_{E}=-0,85\right)$ in extensively used meadow areas (Table 4$)$.

\section{Conclusions}

1. Intensity of sown meadows succession depends upon the character of grassland management. Positive correlation between the number of vascular plant species and sown meadow age $\left(r_{E}=0,95 ; r_{I}=0,59\right)$ revealed that botanical diversity in extensively used grassland restores much faster than under intensive farming conditions.

2. Fertilization of meadow sustains productivity of grassland but impedes the restoration of botanical diversity. In highly productive meadows the restoration of botanical diversity is markedly slower. In grasslands fertilized every year, the sown species consistent with the existing ecological conditions are more competitive; they get enough nutrients, and, therefore, suppress slower growing and less demanding plants.

3. Restoration of semi-natural meadow ecosystems (especially under conditions of extensive use) by naturalization of sown meadows involves exceptional advantages in comparison with other meadow restoration methods being comparatively cheap, undemanding for large investments and labour expenditures. The approach does not demand complete suspension of economic activity and meadow exploitation and reduces possibility for unwanted plant species to establish.

\section{Acknowledgement}

Sincere thanks to Mrs. J. Miklušienè and Mrs. V. Ptašekienè for their kind linguistic advices. 


\section{References}

1. Eringis, K. Perennial cultivated pastures of Lithuania (Долголетние культурные пастбища Литвы). Vilnius: Institute of Botany, 1964. 502 p. (in Russian).

2. Green, B. H. Agricultural intensification and the loss of habitat, species and amenity in British grasslands: a review of historical change and assessment of future prospects. Grass and Forage Science, Vol 45. Blackwell Publishing, 1990, p. 365-372.

3. Muller, S.; Dutoit, T.; Alard, D.; Grévilliot, F. Restoration and rehabilitation of species-rich grassland ecosystems in France: a review. Restoration Ecology, Vol 6. Blackwell Publishing, 1998, p. 94-101.

4. Sendžikaitè, J. Perennial changes in extensively used sown meadow communities. Botanica Lithuanica, Vol 8, No 3. Vilnius: Botanikos instituto leidykla, 2002, p. 261276.

5. Pywell, R. F.; Bullock, J. M.; Hopkins, A.; Walker, K. J.; Spars, T. H.; Burke, M. J. W.; Peel, S. Restoration of species-rich grassland on arable land: assessing the limiting processes using a multi-site experiment. Journal of Applied Ecology, Vol 39. British Ecological Society, 2002, p. 294-309.

6. Bakker, J. D.; Wilson, S. D.; Christian, J. M.; Li, X.; Ambrose, L. G.; Waddington, J. Contingency of grassland restoration on year, site, and competition from introduced grasses. Ecological Applications. Ecological Society of America, Vol 13, 2003, p. 137-153.

7. Hellström, K.; Huhta, A.-P.; Rautio, P.; Tuomi, J.; Oksanen, J.; Lain, K. Use of sheep grazing in the restoration of semi-natural meadows in northern Finland. Applied Vegetation Science, Vol 6. Opulus Press, 2003, p. 45-52.

8. Lindborg, R.; Eriksson, O. Effects of restoration on plant species richness and composition in Scandinavian seminatural grasslands. Restoration Ecology, Vol 12. Blackwell Publishing, 2004, p. 318-326.

9. Wilson, S. D.; Bakker, J. D.; Christian, J. M.; Li, X.; Ambrose, L. G.; Waddington, J. Semiarid old-field restoration: is neighbor control needed? Ecological Applications. Ecological Society of America, Vol 14, 2004, p. $476-484$.

10. Sendžikaitè, J.; Pakalnis, R. Naturalization of sown meadow communities and biological diversity restoration in Lithuania. In: The World Conference on Ecological Restoration. Ecological Restoration: A Global Challenge. Conference abstracts, Zaragoza, Spain, 12-18 September, 2005, p. 138. www.ecologicalrestoration.net/archivos/Sendzikaite,J.doc

11. Bakker, J. P.; van Digglen, R. Restoration of dry grasslands and heathlands. In: van Andel J.; Aronson J. (ed.). Restoration ecology: the new frontier. Blackwell Publishing, 2006, p. 95-110.

12. Sendžikaite, J.; Pancekauskienè, D. Structure and productivity of sown meadow communities on the Central Lithu- anian plain (on the model of Graisupis Experimental Field Station). Botanica Lithuanica, Vol 9, No 4. Vilnius: Botanikos instituto leidykla, 2003, p. 261-276.

13. Braun-Blanquet, J. Pflanzensoziologie - Grundzüge der Vegetationskunde (ed. 3). Berlin-Wien-New York: Springer, $1964.865 \mathrm{p}$.

14. Rašomavičius, V. (ed.). Vegetation of Lithuania. 1. Meadows (Lietuvos augalija. 1. Pievos). Kaunas-Vilnius: Šviesa Publishers, 1998. 269 p. (in Lithuanian).

15. Gudžinskas, Z. Vascular plants of Lithuania (Lietuvos induočiai augalai). Vilnius: Institute of Botany, 1999. 212 p. (in Lithuanian).

16. Dylis, N. (ed.). Programme and methods of biogeocenological investigations. Moskow: Nauka, 1974. 401 p.

17. Lapinskienè, $\mathrm{N}$. The underground part of the grassy plants and phytocenoses in the Lithuanian SSR (Подземная часть травянистых растений и фитоценозов в Литовской ССР). Vilnius: Mokslas, 1986. 176 p. (in Russian).

18. Petkevičius, A.; Stancevičius, A. Fodder plants of meadows and pastures (Pašariniai pievų ir ganyklų augalai). Vilnius: Mokslas, 1982.176 p. (in Lithuania).

19. Ellenberg, H. Zeigerwerte der Gefäßpflanzen (ohne Rubus). In: Ellenberg H.; Weber H. E.; Düll R.; Wirth V.; Werner W.; Paulissen D. Zeigerwerte von Pflanzen in Mitteleuropa. 3. Aufl. Scripta geobotanica, Vol 18, 1991, p. 9-166.

20. Magurran, A. E. Ecological diversity and its measurement. Princeton: Princeton University Press, 1988. 192 p.

21. Sakalauskas, V. Statistics with STATISTICA (Statistika su STATISTICA). Vilnius: Margi raštai, 1998. 227 p. (in Lithuanian).

22. Basalykas, A. (ed.) Physical Geography of the Lithuanian SSR. 1 (Lietuvos TSR fizinè geografija. 1).Vilnius: Mintis, 1958. 504 p. (in Lithuanian).

23. Basalykas, A. (ed.) Physical Geography of the Lithuanian SSR. 2 (Lietuvos TSR fizinè geografija. 2).Vilnius: Mintis, 1965. 496 p. (in Lithuanian).

24. Juodis, J. Soil regions (Dirvožemių rajonai). In: Liekis, A. (ed.). Soils of Lithuania (Lietuvos dirvožemiai). Vilnius: Lietuvos mokslas, 2001, p. 698-707 (in Lithuanian).

25. Juodis, J.; Vaičys, M. Land typology (Žemių tipologija). In: Liekis A. (ed.). Soils of Lithuania (Lietuvos dirvožemiai). Vilnius: Lietuvos mokslas, 2001, p. 1025-1045 (in Lithuanian).

26. Losvik, M. H. Phytosociology and ecology of old hay meadows in Hornaland, western Norway in relation to management. Vegetatio, No 78, Springer, 1988, p. 157187.

27. Huhta, A. P. Vegetation changes in semi-natural meadows after abandonment in coastal northern Finland. Nordic Journal of Botany, No 16. Council for Nordic Publications in Botony, 1997, p. 457-472. 


\section{EKSTENSYVUSIS SĖTŲJŲ PIEVŲ NAUDOJIMAS BOTANINEI İVAIROVEI ATKURTI}

\section{J. Sendžikaitè, R. Pakalnis}

\section{Santrauka}

Skirtingo naudojimo pobūdžio (intensyviojo ir ekstensyviojo) sètujų pievų augmenijos bendrijų būklè įvertinta ištyrus 10-14 metų naudotų pievų mezofilinus žolynus (Graisupio stotyje, Kėdainių r.). Palyginus intensyviai ir ekstensyviai naudojamų sėtujų pievų tyrimų duomenis galima teigti, kad žolynų naudojimo pobūdis turi įtakos pievų sukcesijos intensyvumui. Nustatyta teigiama induočių augalų rūšių skaičiaus ir sètujų pievų amžiaus koreliacija leidžia teigti, kad ekstensyviai naudojamame žolyne botaninè rūšių ịvairovė atsikuria žymiai greičiau $\left(r_{E}=0,95\right)$ nei intensyviojo ūkininkavimo sąlygomis $\left(r_{I}=0,59\right)$. Mažas vienodų induočių augalų rūšių, inventorizuotų skirtingo naudojimo žolynuose, kiekis (23 rūšys) lèmė nedidelę Sörensen koeficiento reikšmę $\left(C_{S}=0,42\right)$, patvirtinančią, kad tirtos pievos yra skirtingu sukcesijos stadijų.

Prasminiai žodžiai: sètosios pievos, sukcesija, intensyvusis ir ekstensyvusis naudojimas, produktyvumas, botaninès įvairovès atsikūrimas.

\section{ЭКСТЕНСИВНОЕ ПОЛЬЗОВАНИЕ СЕЯНЫХ ЛУГОВ КАК ВОЗМОЖНОСТЬ ВОССТАНОВЛЕНИЯ БОТАНИЧЕСКОГО РАЗНООБРАЗИЯ}

\section{Ю. Сенджикайте, Р. Пакальнис}

Резюме

Исследовано состояние интенсивно и экстенсивно пользуемых мезофильных сеяных лугов (10-14-е годы пользования; стационар Грайсупис, Литва). Сравнение результатов исследования сеяных лугов различной интенсивности пользования позволяет утверждать, что характер пользования травостоев оказывает влияние на интенсивность сукцессий лугов. Установленная положительная корреляция количества видов сосудистых растений и возраста сеяного луга показывает, что в экстенсивно используемом травостое ботаническое разнообразие восстанавливается значительно быстрее $\left(r_{E}=0,95\right)$, чем в условиях интенсивного хозяйствования $\left(r_{I}=0,59\right)$. Сравнительно небольшое количество общих видов сосудистых растений (23 вида) в травостоях различной интенсивности пользования определило небольшое значение коэффициента Sörensen $\left(\mathrm{C}_{S}=0,42\right)$, подтверждающее наличие различных стадий сукцессий исследованных лугов.

Ключевые слова: сеяные луга, сукцессия, интенсивное и экстенсивное пользование, продуктивность, восстановление ботанического разнообразия.

Jūratė SENDŽIKAITĖ. Dr, research worker, Laboratory of Landscape Ecology, Institute of Botany (Vilnius, Lithuania). Doctor of Biomedical Sciences (botany, 04 B), Institute of Botany, 2002. Research interests: ecology, botany.

Romas PAKALNIS. Dr, head of Laboratory of Landscape Ecology, Institute of Botany (Vilnius, Lithuania).

Doctor of Sciences (botany),Vilnius University, 1971. Employment: director of fInstitute of Botany, (1989-2002), senior researcher (1974), engineer of forestry (1963), Lithuanian Academy of Agriculture (now Lithuanian University of Agriculture). Publications: author of 4 monographs, 2 study-guides, 3 scientific issues, over 100 research papers. Honorary awards: Valdas V. Adamkus prize-winner (1996), Medal of the Lithuanian Independence (2000), Order of Gediminas (2001). Probation in Sweden. Research interests: ecology, botany, environment protection, sustainable development, education. 